\title{
Optical Biomedical Diagnostics: Sensors with Optical Response Based on Two-Photon Excited Luminescent Dyes for Biomolecules Detection
}

\author{
V. M. Yashchuk, ${ }^{1}$ S. M. Yarmoluk, ${ }^{2}$ V. Yu. Kudrya, ${ }^{1}$ M. Yu. Losytskyy, ${ }^{1,2}$ V. P. Tokar, ${ }^{1,2}$ V. M. Kravchenko, ${ }^{1}$ \\ V. B. Kovalska, ${ }^{2}$ A. O. Balanda, ${ }^{2}$ and D. V. Kryvorotenko ${ }^{2}$ \\ ${ }^{1}$ Department of Physics, Taras Shevchenko Kyiv University, Build.1, Academic Glushkov's Street 2, Kyiv 03680, Ukraine \\ ${ }^{2}$ Institute of Molecular Biology and Genetics, National Academy of Sciences of Ukraine, Zabolotnogo Street 150, \\ Kyiv 03143, Ukraine
}

Correspondence should be addressed to V. M. Yashchuk, vmyashchuk@univ.kiev.ua

Received 29 February 2008; Accepted 4 April 2008

Recommended by Stoyan Tanev

The spectral properties of novel styryl dyes developed for the biomacromolecules (such as DNA) detection and imaging were investigated. The energy structures of dye molecules were examined. The spectral data prove that dyes aggregate and interact with DNA. The essential increase of the fluorescence intensity of dyes in the presence of DNA was observed. The photostability and phototoxic influence on the DNA of several styryl dyes were studied by analyzing absorption, fluorescence, and phosphorescence spectra of these dyes and dye-DNA systems. Changes of the optical density value of dye-DNA solutions caused by the irradiation were fixed in the DNA and dye absorption wavelength regions. Fluorescence emission of dye-DNA complexes upon two-photon excitation at wavelength $1064 \mathrm{~nm}$ with the 20 -nanosecond pulsed YAG: $\mathrm{Nd}^{3+}$ laser and at $840 \mathrm{~nm}$ with the 90 famtosecond pulsed Ti:sapphire laser was registered. The values of two-photon absorption cross-sections of dye-DNA complexes were evaluated.

Copyright ( 92008 V. M. Yashchuk et al. This is an open access article distributed under the Creative Commons Attribution License, which permits unrestricted use, distribution, and reproduction in any medium, provided the original work is properly cited.

\section{INTRODUCTION}

Fluorescent probes are widely used in biological and medical studies for the detection of biological molecules and fluorescent imaging of biological objects [1]. The two important properties of these probes permitting their application for fluorescent detection are (1) strong affinity of the probe binding to the biological molecule of interest, and (2) sharp enhancement of the fluorescence intensity of the probe upon such binding. Commonly, the fluorescence of the probes is excited with either ultraviolet (UV) or visible light, so that the probe molecule is excited by one absorbed photon (single photon excitation). The single photon excitation (SPE) approach has several disadvantages. First, the light in UV and visual regions (250-700 nm) cannot penetrate deep into the biological tissue, being both absorbed and scattered, and hence is not useful for the tissue fluorescent scanning. The probes with absorption in the region 700-1000 nm, where the majority of biological objects are transparent, are not easy to design, and if designed such probes would fluoresce in infrared (IR) region, which is not convenient for observation. Second, using SPE (especially with UV light) causes significant damage of the studied biological objects, not only in the point of detection, but also on the whole way of the beam. Third, when used for three-dimensional (3D) scanning microscopy, SPE results in rather restricted resolution in the two directions perpendicular to the beam and in very poor resolution along the direction parallel to the beam propagation.

The problems mentioned above could be overcome with the help of two-photon excitation (TPE) of the fluorescent probe. By TPE, which is a nonlinear process, one probe molecule is excited by two photons absorbed simultaneously (or, to be more precise, in a very short period of time). Hence, the TPE efficiency is proportional to the square of the excitation beam intensity, which permits the localization of the excitation only at the focusing point, and not on the whole way of the beam. Moreover, the wavelength of the exciting beam for TPE is about 2 times higher than this for SPE beam for the same probe, which permits excitation 
of visual fluorescence in the transparency region of biological objects (700-1000 nm), besides, the higher excitation wavelength means the lower scattering of excitation beam. Thus, using two-photon excitation of fluorescent probes in procedures of biological objects detection permits deeper penetration of exciting beam into the tissue, excitation of visual fluorescence in near infrared spectral region, where the majority of biological objects are transparent, decreased photodamage of the studied object, and obtaining of wellresolved three-dimensional image of biological object $[2,3]$.

The efficiency of TPE of a molecule at the constant excitation intensity is determined by the value of twophoton absorption cross-section. The higher the value of two-photon absorption cross-section is, the lower excitation intensity is required to obtain the same fluorescence intensity. And lower excitation intensity means (1) cheaper and simpler equipment needed and (2) lower photodamage of the sample. Thus, the successful application of the TPE for the detection of biological molecules requires the fluorescent probes with (1) high affinity to the biological molecules of interest, and (2) high value of the two-photon absorption cross section. Unfortunately, such probes are not developed at all for the moment.

It is important that the luminescent biomedical probesensor could be applied to the study of the living cells (e.g., with the fluorescent microscopy). It was shown in a number of papers that the widely used fluorescent probes photochemically destroy the biological objects (DNA, RNA, etc.). So probes have to be nonphototoxic. Besides, the probe should be photostable so that the biomolecule could be studied for the long enough period of time without the probe damage. The phototoxic influence of the dye molecule on the DNA or RNA can take place either directly via the excitation energy transfer from the dye to the nucleotide bases or indirectly via the third molecule (e.g., by the triplet excitation energy transfer to the oxygen molecule resulting in the generation of the toxic singlet oxygen $[4,5])$. It was shown [6-15] that the absorption bands (connected with the first electronic transition) of the DNA, RNA, and nucleotide bases are located in the near UV spectral region with the maximum near $260 \mathrm{~nm}$. At the same time, the corresponding absorption bands of the majority of dyes used as luminescent probes are located in the visual spectral region $(>400 \mathrm{~nm})$ [1]. The fact that the first excited singlet and triplet energy levels of dyes are situated essentially lower than correspondent levels of any nucleotides does not allow the excitation energy transfer from the dye to the DNA thus making impossible the direct phototoxic influence of the dye on the DNA. Nevertheless, the molecules such as porphyrines or other pigments that destroy the DNA indirectly by the generation of singlet oxygen are well known and used in the photodynamic therapy.

In the presented work we study the styryl dyes as the probes for the TPE fluorescent detection of DNA. Styryl dyes are known as having high two-photon absorption crosssection values [2, 16]; one of the benzothiazolium styryls was shown to give strong fluorescence upon TPE by $1064 \mathrm{~nm}$ irradiation [17]. On the other hand, significant fluorescent response on the dsDNA presence is observed for these dyes [18-21]. Recently series of monomer and homodimer benzothiazolium styrylcyanines was synthesized and studied for their efficiency as TPE nucleic acids sensitive dyes. It was shown that dyes modified with spermine-like linkage/tail group demonstrate increased sensitivity to DNA [19]. They have low intrinsic emission intensity and enhance their fluorescence intensity up to three orders of magnitude in presence of DNA. We believe that the influence of sperminelike linkage/tail group on the dye sensitivity to DNA is due to the additional interaction of the positively charged nitrogen atom with the DNA. Complexes of studied dyes with DNA also demonstrate intensive emission upon the TPE [19].

In the presented work, two-photon excited fluorescence of monomer and homodimer styrylcyanine dyes based on benzothiazole, naphtathiazole, benzoimidazole, pyridinium, quinoline, and 4-oxo-thieno[2,3-d]pyrimidinium heterocyclic residues and containing charged spermine-like linkage/tail groups was studied in the presence of DNA. Besides, for several dyes the fluorescent and phosphorescent properties at low temperature, as well as photochemical stability and phototoxicity to the DNA, were investigated.

\section{METHODOLOGY}

The total DNA from chicken erythrocytes was purchased from Sigma-Aldrich Inc (St. Louis, Mo, USA). The dye DstMdO (Figure 1) was obtained by the boiling of quaternary salt and $p$-dimethylaminobenzaldehyde in acetic anhydride. Dyes Bos-1, DBos-21, DBos-24, DBos-25, DBos-28, and DBos-30 (Figure 1) were obtained as described in [19]. Dyes S-3, S-20, S-33, S-45, and S-46 (Figure 1) were obtained as described in [21]. Dyes F, Sbt, Sil, Dst-6, Dst-10, Dbt10 , and Dil-10 (Figure 1) were synthesized according to the procedures described in [18]. The synthesis of dyes S48, Tio-1, and S-40 (Figure 1) was described in [20]. The structures of the dyes were confirmed with $\mathrm{H}^{1} \mathrm{NMR}$ and element analysis.

The samples were prepared in distilled water and $0.05 \mathrm{M}$ TRIS-HCl buffer, $\mathrm{pH}$ 8.0. The concentrations of dye and DNA were, respectively, $10^{-5} \mathrm{M}$ and $6 \times 10^{-5} \mathrm{M}$ b.p. (base pairs) for the absorption measurements and photodamage experiment, and $10^{-4} \mathrm{M}$ and $6 \times 10^{-4} \mathrm{M}$ b.p., respectively, for the low-temperature luminescence measurements. For the low-temperature measurements, the prepared solutions were poured out into the special cell so that the upper surface is open, and then frozen at the temperature of the boiling nitrogen $(77 \mathrm{~K})$. The excitation beam was directed to the open surface, and from the same surface the luminescence was registered.

The steady state fluorescence and phosphorescence measurements were performed using laboratory-designed equipment; absorption spectra were recorded with the help of a Specord UV-VIS spectrophotometer. The photodamage of dyes and the DNA+dye systems was performed by exposition of the corresponding solution to the visible spectrum irradiation of the $1 \mathrm{~kW} \mathrm{Hg-lamp.}$

The measurements were carried out at $77 \mathrm{~K}$ and ambient temperatures. 


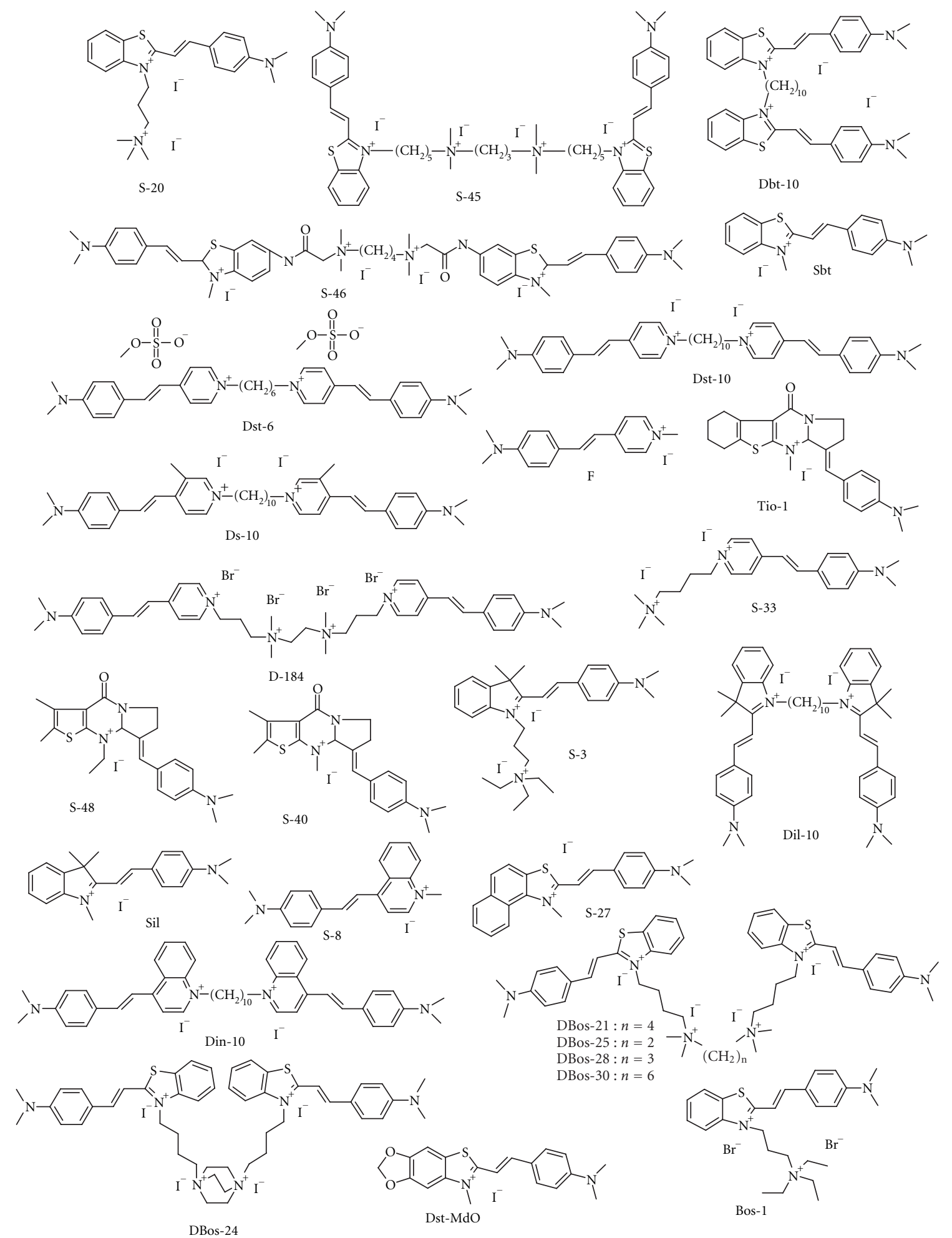

FIgURE 1: Chemical structures of the studied dyes.

The TPE fluorescence measurements of the dyes in DNA presence were performed by using the $1064 \mathrm{~nm}$ irradiation of the 20 nanoseconds pulsed YAG: $\mathrm{Nd}^{3+}$ laser as the excitation source. Experimental setup was used and two-photon absorption cross-section values calculation performed as described in [19].

During the TPE fluorescence measurements at wavelength $840 \mathrm{~nm}$, a Ti:Sapphire laser (Mira Optima 900-F, 
Coherent Inc (Santa Clara, Calif, USA) pumped up with the cw Nd: $\mathrm{YVO}_{4}$ laser (Coherent) and generating $90 \mathrm{fs}$ $\operatorname{cosec}^{2}$-shaped pulses with repetition rate $76 \mathrm{MHz}$ was used. Parameters of laser were monitored by autocorrelator (APE Autocorrelator mini) and power meter (Field Master GS, LM-10, Coherent). The fluorescence light was detected at right angle as it passed the telecentric system of two lenses and the entrance slit $(100 \mu)$ of the spectrograph $(500 \mathrm{~mm}$ Imaging Spectrograph SP-2558, Princeton Instruments Acton (Acton, Mass, USA)). A CCD camera (CCD-Spec10:256E/TEPLUS $1024 \times 256$ Open-electrode, Marconi CCD 30-11) was used as a detector of TPE-fluorescence.

\section{RESULTS AND DISCUSSION}

\subsection{The study of SPE and TPE fluorescence of dyes and dye-DNA complexes}

Styryl dyes are known to be efficiently excited via the TPE $[2,16,17]$. Thus, the study of the styryl dyes as possible fluorescent probes for the DNA was performed by us as follows. First, wide series of styryl dyes based on different heterocyclic residues were synthesized and studied as the SPE fluorescent probes for DNA detection. Second, the dyes demonstrating both high value of fluorescence intensity in DNA presence and sharp emission intensity increase upon binding to DNA were studied in the TPE experiment.

Recently, the series of novel monomer and homodimer styrylcyanine dyes based on benzothiazole, naphtathiazole, benzoimidazole, pyridinium, quinoline, and 4-oxo-thieno (2,3-d) pyrimidinium heterocyclic residues (Figure 1) was elaborated, synthesized, studied, and partly reported [18, 20, 21]. The dyes demonstrated high DNA sensitivity, namely, fluorescence intensity of dyes upon SPE enhanced up to thousand times upon binding with DNA (Figure 2). The study of the dyes absorption and fluorescence spectra at room temperature in water buffer solution as well as in the DNA presence revealed that the spectra of majority of the studied dyes are connected with the absorption and fluorescence of the separate styryl chromophores. Meanwhile, for the styryl homodimer dyes containing the spermine-like linker (DBos-21, DBos-24, DBos-25, DBos-28, DBos-30, S45 , and $\mathrm{S}-46)$ the band corresponding to the aggregate of the styryl chromophores is dominating in the absorption spectrum of the free dyes, while the addition of the DNA to the solution leads to the decrease of the aggregate band and growth of the monomer ones. At the same time, the mentioned aggregates were found to be nonfluorescent at room temperature, thus the fluorescence spectra of the mentioned dimers, as well as of all the studied dyes (both free and bound to DNA), correspond to the emission of the separate styryl chromophores [19]. Hence, the strongly enhanced fluorescence of the studies styryls in DNA presence is the emission of the styryl chromophores, bound to the DNA molecule. The fixation of the separate chromophore on DNA (via either intercalation or groove-binding) leads to the decrease in the rate constant of the nonfluorescent deactivation of the singlet electronic excitation of the dye,

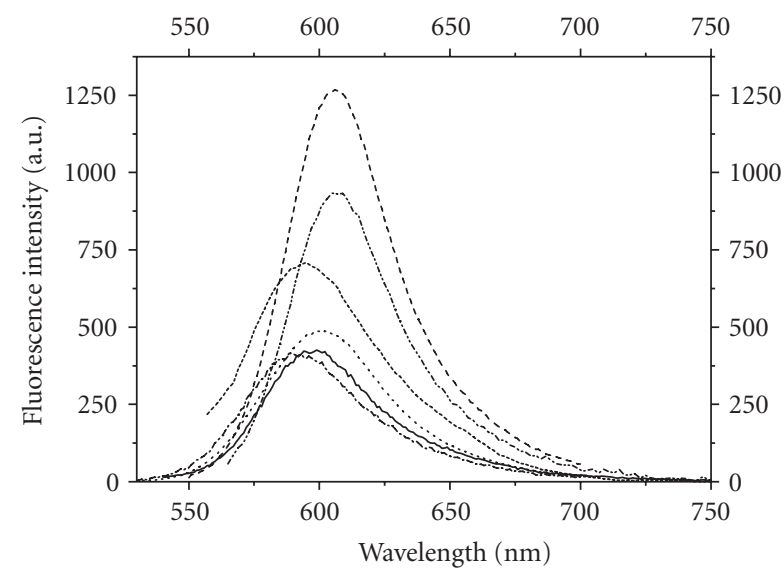

$$
\begin{aligned}
& \text {-.... Sbt in buffer }(\times 20) \\
& \text { Sbt in DNA presence } \\
& \text { - Bos-1 in buffer }(\times 20) \\
& \text { - - - Bos-1 in DNA presence } \\
& \text {....... DBos-28 in buffer }(\times 800) \\
& \text {-...... DBos-28 in DNA presence }
\end{aligned}
$$

FIGURE 2: SPE fluorescence spectra of dyes in free state and in DNA presence.

and thus to the increase of the dye fluorescence quantum yield [22].

In order to enhance the dye-to-DNA affinity, some of the studied dyes contained the spermine-like tail or linking group. It was shown by us earlier [23] that the sperminelike tail group enhances the constant of dye to DNA binding equilibrium $\left(K_{b}\right)$ in about three times $\left(1.8 \times 10^{4} \mathrm{M}^{-1}\right.$ for Sbt and about $6 \times 10^{4} \mathrm{M}^{-1}$ for $\left.\mathrm{S}-20\right)$. We believe this enhancement to be connected with the additional interaction of the charged spermine tail group with the DNA. It should be mentioned that the cyanine dyes sensitive to DNA possess the $K_{b}$ values of the same order of magnitude as the benzothiazole styryl dyes Sbt and S-20 [24], though for some of the cyanines (e.g., TO) the value of $K_{b}$ was reported to be about $10^{6} \mathrm{M}^{-1}[25]$.

\subsection{The effect of dyes aggregations on their phosphorescent properties}

Phosphorescence excitation and emission spectra of benzothiazole homodimer dyes solutions were measured (Figure 3). The energy of the lowest triplet level of dyes is about $1.85 \mathrm{eV}\left(14900 \mathrm{~cm}^{-1}\right)$. This value is less than the value of the lowest triplet level among DNA bases thus the triplet electron excitation energy transfer from DNA to the dye can take place and is still under study. The phosphorescence lifetime was evaluated and amounted about 20 milliseconds.

Fluorescence excitation and emission spectra of benzothiazole homodimer dyes solutions at liquid nitrogen temperature were obtained. Also fluorescence of dyes' aggregates at liquid nitrogen temperature was registered while at room temperature these aggregates are virtually nonfluorescent. As far as fluorescence excitation spectra of aggregates coincide with phosphorescence excitation spectra of dyes, it was 


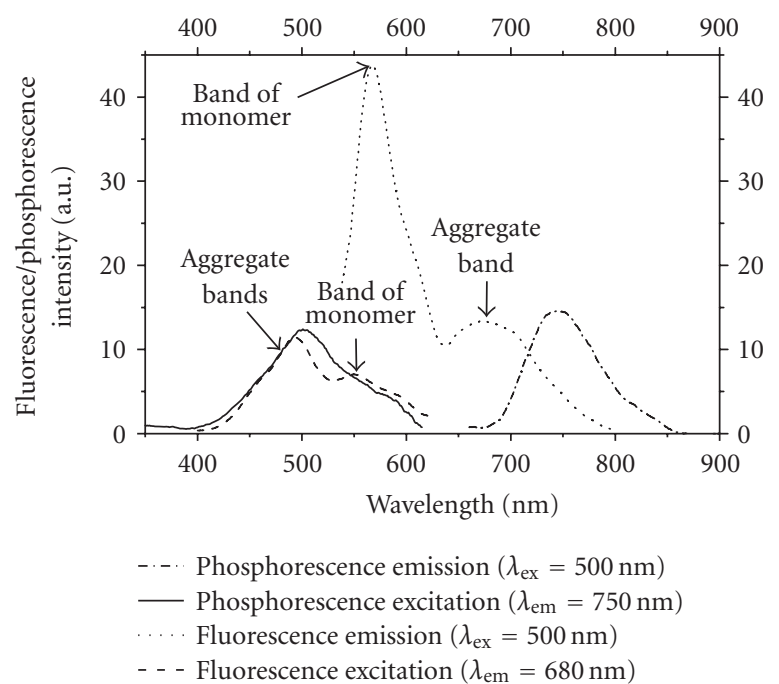

FIGURE 3: Fluorescence and phosphorescence emission and excitation spectra of DBos-30 water solution at liquid nitrogen temperature $\left(C=10^{-4} \mathrm{M}\right)$.

concluded that, namely, aggregates are mainly responsible for the phosphorescence (Figure 3). In order to exclude the interaction between the dye-DNA complex and the molecular oxygen in the water solution the studying of the dyes emission in vacuum is under research now.

\subsection{TPE fluorescence of dyes and dye-DNA complexes}

The TPE study of the fluorescent properties of the mentioned styryl dyes in the presence of DNA was performed.

The dye-DNA complexes revealed intensive emission upon two-photon excitation (TPE) at wavelength $1064 \mathrm{~nm}$ with the 20 nanoseconds pulsed YAG: $\mathrm{Nd}^{3+}$ laser. Here the numerical results of dyes' studying upon the TPE in complexes with DNA are presented. The values of dyes' twophoton absorption (TPA) cross section $\delta$ were evaluated (see Table 1). The calculations of $\delta$ were performed using the respective value for Rhodamine 6G [26]. As it is seen from Table 1, the $\delta$ values of dye-DNA complexes are about $10^{-50} \mathrm{~cm}^{4} \mathrm{~s}$.

Dyes from the series with each type of chromophore in complexes with DNA demonstrated emission upon TPE at $840 \mathrm{~nm}$ with the 90 femtosecond pulsed Ti:sapphire laser (see Figure 4). TPA cross sections $\delta$ at wavelength $840 \mathrm{~nm}$ are under evaluation now.

The possibility of the dye DBos-21 application for the TPE fluorescent cell staining was studied. HeLa cells were incubated for 1 hour with DBos-21 $(5 \mu \mathrm{M})$ and imaged using $880 \mathrm{~nm}$ excitation of the femtosecond pulsed Ti:Sapphire laser (Tsunami, Spectra Physics). TPE fluorescent image of the cells stained with DBos-21 is shown in Figure 5.

The TPE fluorescent imaging study shows that the styrylcyanine dimer dye DBos-21 containing the sperminelike linking group successfully permeates through the living cell membrane. Besides, this dye demonstrated mostly mitochondria staining, possibly because of high positive charge of the dye.
TABLE 1: The values of TPA cross section $\delta$ of dyes in DNA presence upon excitation at wavelength $1064 \mathrm{~nm}$.

\begin{tabular}{cccc}
\hline Dye & $\delta, 10^{-50} \mathrm{~cm}^{4} \cdot \mathrm{s}$ & Dye & $\delta, 10^{-50} \mathrm{~cm}^{4} \cdot \mathrm{s}$ \\
\hline S-20 & 2.3 & S33 & 1.1 \\
Bos-1 & 2.2 & D-184 & 1.1 \\
DBos-21 & 1.7 & S40 & 0.2 \\
DBos-24 & 1.4 & S48 & 0.2 \\
DBos-25 & 1.7 & Tio1 & 0.2 \\
DBos-28 & 2.1 & Sil & 1.7 \\
DBos-30 & 1.8 & Dil-10 & 1.0 \\
S45 & 1.4 & S3 & 0.9 \\
S46 & 0.3 & S8 & 0.4 \\
Sbt & 1.7 & Din-10 & 0.4 \\
Dbt10 & 0.3 & S27 & 0.8 \\
F & 0.04 & Rhodamine 6G & 5.5 \\
Dst-6 & 0.6 & & \\
Dst-10 & 0.6 & & \\
Ds-10 & 0.4 & & \\
\hline
\end{tabular}

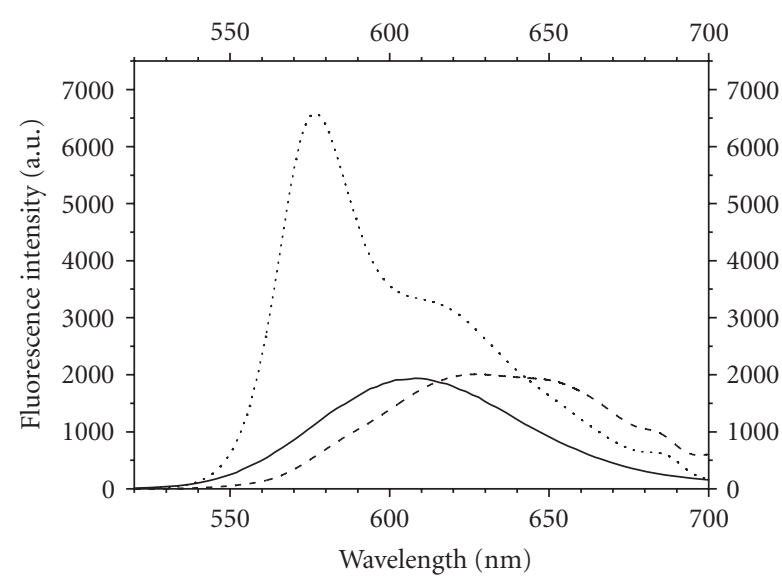

$$
\begin{aligned}
& \text { Upon TPE }\left(\lambda_{\mathrm{ex}}=840 \mathrm{~nm}\right) \text { : } \\
& -- \text { - S33-DNA complexes in water buffer } \\
& \ldots . \text { Rhodamine B in ethanol } \\
& \text { Upon SPE }\left(\lambda_{\mathrm{ex}}=506 \mathrm{~nm}\right) \text { : } \\
& \quad \text { S33-DNA complexes in water buffer (normalized) }
\end{aligned}
$$

FIGURE 4: Fluorescence emission spectra of dye-DNA water buffer solution and Rhodamine B ethanol solution upon SPE and TPE (concentration of dyes $C=1.5 \times 10^{-5} \mathrm{M}$ ).

Thus, the styrylcyanine dimmer dyes containing the spermine-like linking group could be successfully applied for the TPE fluorescent imaging of the living cells.

\subsection{The study of dyes phototoxicity and photostability}

\subsubsection{The first excited singlet and triplet levels of dyes}

For the use of the dyes as biomedical sensors for the DNA (or RNA) detection and imaging in the study of the living objects the phototoxic influence of these dyes realized via 
TABLe 2: Positions of singlet (S) and triplet (T) levels of the dyes in presence of the DNA, $\mathrm{cm}^{-1}$.

\begin{tabular}{|c|c|c|c|c|c|c|}
\hline Compound & Bos-1 & DBos-21 & DBos-24 & DBos-25 & DBos-28 & DBos-30 \\
\hline S (293 K) & 18250 & 18180 & 18120 & 18120 & 18280 & 17940 \\
\hline S (77 K) & 19100 & 18800 & 19530 & 19160 & 18680 & 19110 \\
\hline $\mathrm{T}(77 \mathrm{~K})$ & 14790 & 14820 & 14240 & 14730 & 14750 & 14890 \\
\hline
\end{tabular}

TABLE 3: Positions of singlet $\left(\mathrm{S}_{1}\right)$ and triplet $\left(\mathrm{T}_{1}\right)$ levels of the nucleotides, $\mathrm{cm}^{-1}$.

\begin{tabular}{ccccc}
\hline Compound & dCMP & dGMP & dTMP & dAMP \\
\hline $\mathrm{S}_{1}$ & 33090 & 33030 & 33530 & 34490 \\
$\mathrm{~T}_{1}$ & 26630 & 26320 & 26160 & 25950 \\
\hline
\end{tabular}

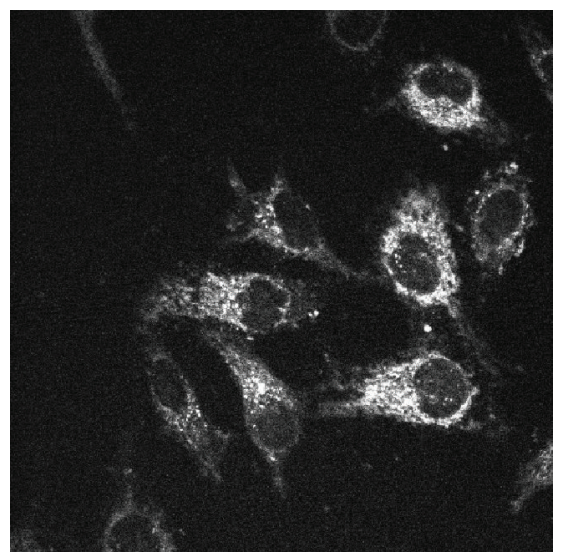

FIGURE 5: TPE fluorescent image of the HeLa cells stained with the dye DBos-21. Fluorescent emission excited with the femtosecond pulsed Ti:Sapphire laser, excitation wavelength $880 \mathrm{~nm}$.

the excitation energy transfer from the dye to the DNA has to be absent. To avoid these processes the first excited singlet and triplet levels of dyes must be situated lower than correspondent levels of any nucleotide. To check this the optical absorption, fluorescence, and phosphorescence spectra of the investigated dyes Bos-1, DBos-21, DBos-24, DBos-25, DBos-28, and DBos-30 as well as correspondent dye+DNA systems were studied. Some of these spectra (namely, for Bos-1, DBos-24 and DBos-30 dyes) are given in Figure 6.

The absorption spectra of the dye Bos-1 both in the presence and the absence of the DNA in visible spectral region consist of the single band with the maxima near 17540 and $18470 \mathrm{~cm}^{-1}$, respectively (Figure 6(a); curves 1,2). Since this band is the most long-wave band in the visible spectral region and the corresponding fluorescence spectrum (Figure 6(a); curves 3,4) obeys the mirror symmetry rule respectively to this absorption band, the latter should be attributed to the absorption transition to the first excited singlet level of the dye molecule. The shift between the maxima of the free dye and the DNA+dye system spectra points to the dye interaction with the DNA. In the absorption spectra of the dimer dyes DBos-24 and DBos-30 in presence of the
DNA the same band with the maximum near $18000 \mathrm{~cm}^{-1}$ (Figure 6(b); curve 2) and $17750 \mathrm{~cm}^{-1}$ (Figure 6(c); curve 2 ), respectively, could be observed. At the same time, the spectrum of DBos-24 in the DNA presence contains one more band with the maximum at $22500 \mathrm{~cm}^{-1}$ shifted to the short-wavelength region relatively to the main band. This short-wavelength band with the maxima at 22200 and $20800 \mathrm{~cm}^{-1}$, respectively, dominates in absorption spectra of both DBos-24 and DBos-30 in absence of the DNA (Figures 6(b); curve 1, and 6(c); curve 1). Taking into account all the above-mentioned results, the short-wavelength band could be attributed to the aggregates of the styryl chromophore. Since for the dimer dyes the tendency to form aggregates is much higher than for corresponding monomers [27], the short-wavelength band is present in the spectra of DBos-24 and DBos-30 in free state, but is absent in the spectrum of Bos-1 containing the same chromophore. At the same time, interaction of dimer dyes with the DNA at low dye to the DNA concentrations ratio generally leads to the fixation of separate chromophores on the DNA molecule, thus resulting in decrease in the aggregates concentration in solution [27]. This explains the decrease in the short-wavelength band contribution to the absorption spectrum of DBos-24 in presence of the DNA, as well as the disappearance of this band in the same spectrum of DBos-30.

The fluorescence of the dyes Bos-1, DBos-24, and DBos30 , both in free form and in DNA presence, at the room temperature was measured in [19]. The maximum of these spectra was situated near $17000 \mathrm{~cm}^{-1}$ similarly to the spectra presented at Figures 6(a), 6(b), 6(c) (curves 3,4). It was shown in [19] with the help of fluorescence excitation spectra that the fluorescence band with the maximum near $17000 \mathrm{~cm}^{-1}$ belongs to the emission of the single styryl chromophores. Thus, the absorption and fluorescence spectra of the dyes Bos-1, DBos-24, and DBos-30 in DNA presence could be used for the calculation of the position of the singlet electronic excited energy level of the nonaggregated chromophore of these dyes, bound to DNA.

The positions of the first excited singlet level of the investigated dyes in nonaggregated form in the presence of the DNA were obtained by intersection of the absorption and fluorescence spectra curves and given in Table 2 .

The values of singlet and triplet energy levels of the nucleotides obtained by us in [28, 29] are presented in Table 3. The comparison of these data with data given in Table 2 removes all doubts that even the triplet level of any nucleotide is situated much upper than the singlet level of any investigated dye. That is why the irradiation of the dye in the visual spectral region exciting the first singlet electronic 

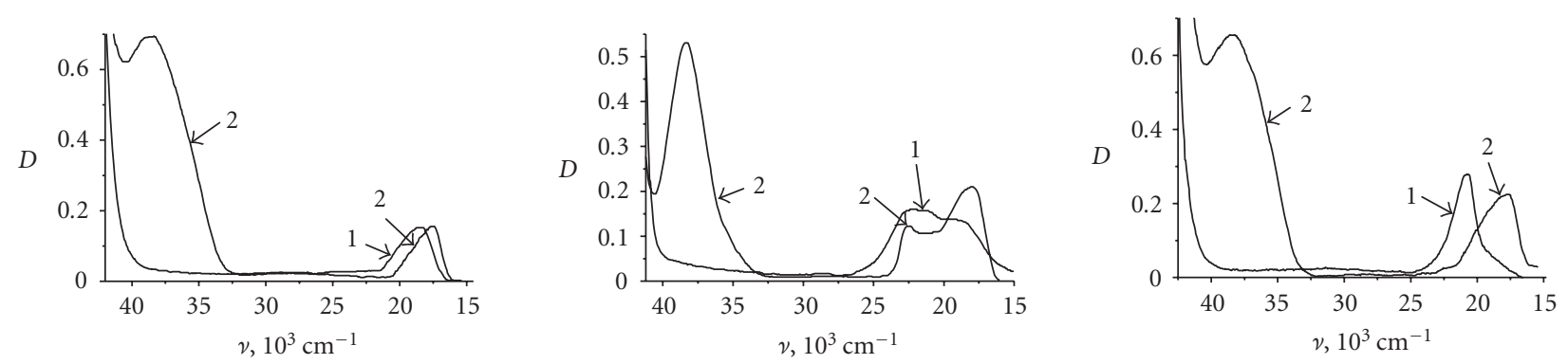

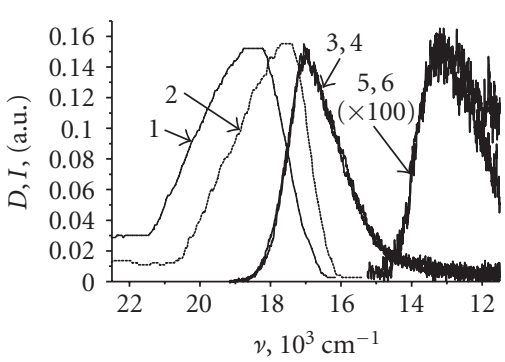

(a)

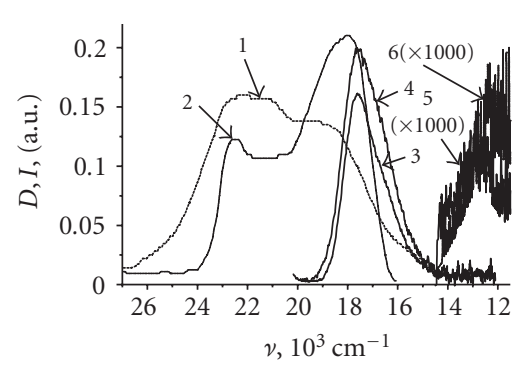

(b)

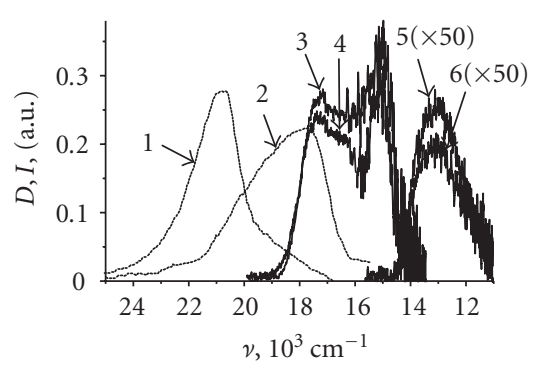

(c)

Figure 6: The absorption $(1,2)$, fluorescence $(3,4)$, and phosphorescence $(5,6)$ spectra $(3-6$ at $\mathrm{T}=77 \mathrm{~K})$ of free styryl dyes $(1,3,5)$ and correspondent systems DNA+dye $(2,4,6)$ of: (a) styryl monomer Bos-1, (b) dimer DBos-24, and (c) dimer DBos-30.

level of the dye cannot be resulted in the excitation energy transfer from the dye to the DNA.

\subsubsection{Effect of irradiation of dyes and the DNA+dye systems on their optical properties}

It is known that the phototoxic influence of a luminescent dye probe on the DNA results in the spectral response of the DNA+dye system. This response is connected with the DNA macromolecule damage and is observed as the change of the optical density of the DNA absorption band (i.e., situated at $260 \mathrm{~nm}$ and corresponds to the $\mathrm{S}_{0} \rightarrow \mathrm{S}_{1}$ electronic transition) in the DNA+dye system under excitation in the dye absorption band (i.e., situated at 400-500 nm and corresponds to its $S_{0} \rightarrow S_{1}$ transition). Besides, the investigation of the optical density changes of the dye band under the free dye excitation at the wavelength of the same band is the way to study the dye photostability. The fact that the DNA absorption band connected with $S_{0} \rightarrow S_{1}$ transition is located far from the same band of majority of dyes and the value of optical density of the absorption band connected with the dye $\mathrm{S}_{0} \rightarrow \mathrm{S}_{2}$ transition (located near $260 \mathrm{~nm}$ ) is much less than corresponding value of the DNA band gives the ground to study the dynamics of the absorption bands optical density changes almost independently for a dye and for the DNA. In our paper, the investigations results of the absorption spectra of the elaborated styryls and the respective DNA+dye complexes (that were recorded with the aim to study both the dye photostability and its phototoxicity influence on the DNA) during the irradiation of the investigated compounds solutions by visible light of $1 \mathrm{~kW} \mathrm{Hg-lamp} \mathrm{were} \mathrm{described.}$

As it is shown in Figure 7 the gradual decrease of $D$ of the absorption spectra bands is observed for free dyes under the irradiation time increase (curve 1). This phenomenon is connected with the damage of dyes $\pi$ electron systems under irradiation and is the evidence that Bos-1 and DBos-30 are not photostable in free form. The gradual decrease of $D$ of the dyes absorption spectra bands in the DNA presence takes place too but essentially slower than for free dyes. Moreover, the decrease rate of $D$ of the DNA+DBos-30 system (Figure 7(b); curve 2) is lower than of the system DNA+Bos-1 (Figure 7(a); curve 2). It means that dimer DBos-30 bound to the DNA is more photostable than monomer Bos-1 bound to the DNA. Comparing the dependencies of optical density on irradiation time of investigated styryl dyes with the same dependence of thiazole orange (TO) dye (Figure 7, curve 4), it is shown that both Bos-1 and DBos-30 dyes (bound to the DNA) are more photostable even than TO dye (bound to the DNA). The rise of the dyes photostability under binding to the DNA occurs, in our opinion, because of the formation of the tough spatial fixation of a dye molecule after the intercalation of this dye in the DNA macromolecule. As the result, the DNA macromolecule screens the dye molecule against external influences and decreases the contacts between the dye molecules and dissolved molecules of oxygen. The last is transferred into the singlet excited electronic state under the direct excitation of the dye and becomes an active oxidant [30]. The results obtained in this paper agree with the data obtained in [31] on investigations of the cyanine dyes. Since the constant of binding to the DNA for the dimer is often higher than for the monomer with the same $\pi$-electron system $[32,33]$, the number of dye molecules bound with the DNA is higher for the dimer DBos-30 than for the monomer Bos-1. Thus the DNA macromolecule screens the dimer molecules rather than the monomer molecules that cause the 


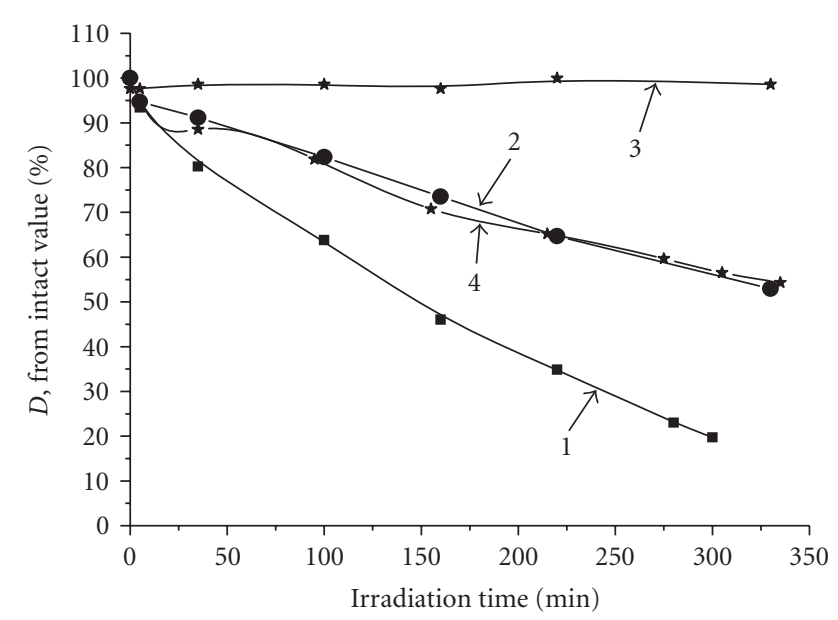

(a)

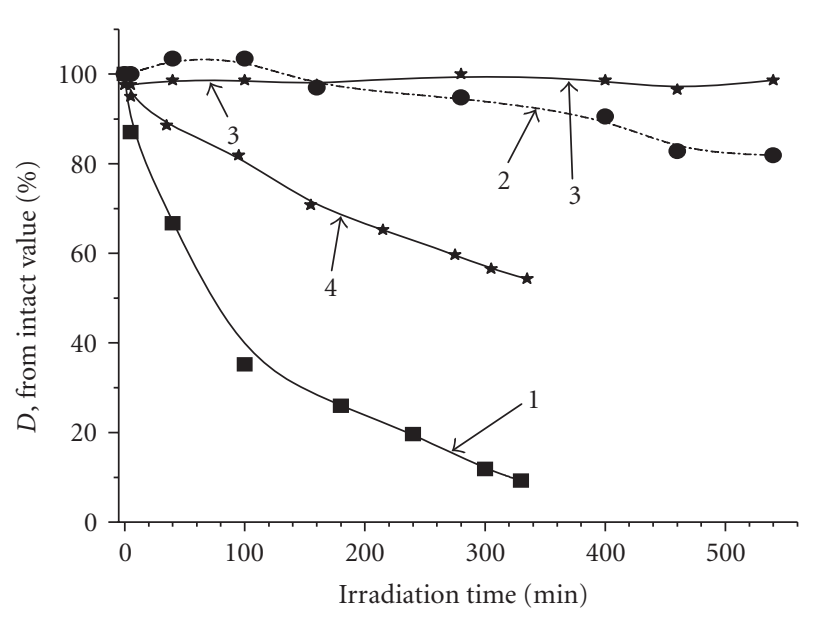

(b)

FIGURE 7: The dependence of optical density (obtained from optical absorption spectra) on irradiation time of dyes: (a) Bos-1 and (b) DBos-30 (1-free dye, 2-DNA+dye, 3-the DNA maximum $260 \mathrm{~nm}, 4$ - TO with the DNA).

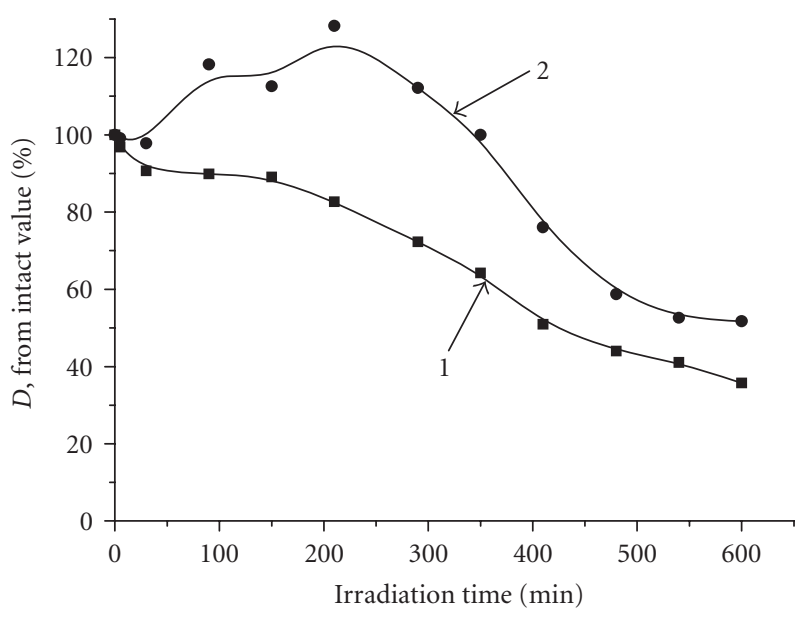

(a)

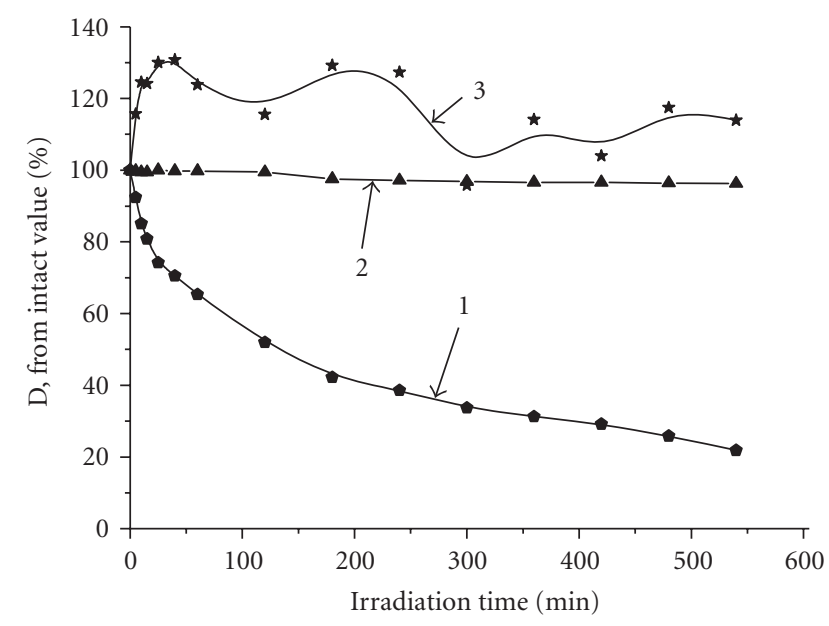

(b)

FIGURE 8: The dependence of optical density (obtained from optical absorption spectra) on irradiation time of dimer dye DBos-24: (a) free dye, (b) bond to the DNA (1-at $23100 \mathrm{~cm}^{-1}, 2$ - at $18500 \mathrm{~cm}^{-1}, 3$ - the DNA maximum at $260 \mathrm{~nm}$ ).

difference in Bos-1 and DBos-30 photostability in the DNA presence.

The dependencies of the DNA band maximum optical density $D$ on time of dye irradiation (Figure 7; curve 3) for the DNA+Bos-1 and DNA+DBos-30 systems were investigated with the aim to study phototoxic influence of the investigated dyes on the DNA. According to our experimental data the value of the optical density $D$ of the investigated dyes absorption band connected with the $S_{0} \rightarrow S_{2}$ transition is changed negligible during all the time of irradiation. As it is shown in Figure 7 the value of $D$ is changed within $3 \%$ at $260 \mathrm{~nm}$ out of its intact value that may be connected with these negligible changes of the dyes $S_{0} \rightarrow S_{2}$ band and experimental errors. It is known that the DNA destruction is either the double strand untwisting or the strand cutting that is reverse to the hypochromic effect, the complete untwisting resulting in the $37 \%$ increase in $D$ value at $260 \mathrm{~nm}$ [34]. That is why the $3 \%$-changes obtained by us cannot be connected with the significant DNA damage and can be included in experimental errors.

So, Bos-1 and DBos-30 dyes are nonphototoxic for the DNA as the first approximation and can be used as the optical biomedical sensors for the DNA detection and imaging. On the other hand, the DNA protects these dyes against the photodamage.

As is shown in Figure 6(b), the dimer DBos-24 absorption band consists of two bands. Comparing Figures 6(a) and $6(\mathrm{~b})$ it can be seen that the long-wavelength band $\left(18500 \mathrm{~cm}^{-1}\right)$ is located close to the monomer Bos-1 band and corresponds to nonaggregated state. The short-wave band $\left(23100 \mathrm{~cm}^{-1}\right)$, in our opinion, is connected with aggregates (the similar case was observed for cyanine dyes 


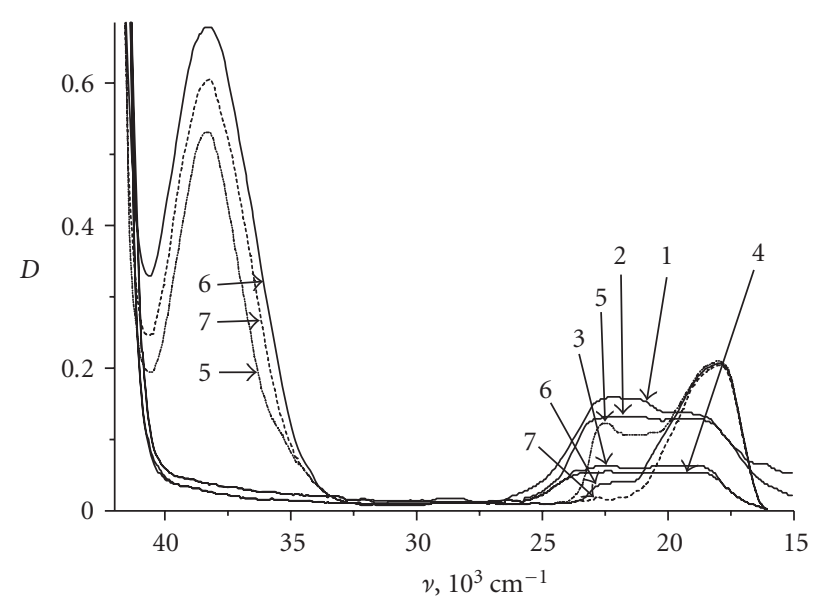

FIgURE 9: The absorption spectra of dimer DBos-24 during irradiation (free dye: 1-intact, 2-210 minutes, 3-540 minutes, 4-600 minutes; dye with DNA: 5-intact, 6-240 minutes, 7-540 minutes).

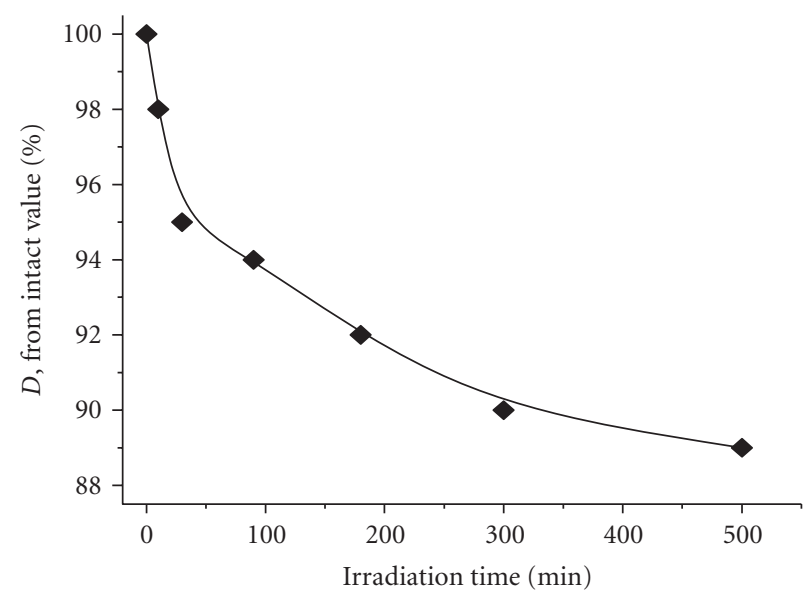

Figure 10: The dependence of optical density of the DNA maximum in the DNA+Dst-MdO system.

$[35,36])$. Using optical absorption spectra of DBos-24 dye and the DNA+DBos-24 system, the dependencies of the optical density $D$ on irradiation time for these molecular systems are obtained (Figures $8(a), 8(b)$ ). The aggregates band of the free dye decreased gradually during all the irradiation time while the nonaggregated dyes band increased slightly at first (Figure 9). In our opinion, it is connected with the fact that aggregates are damaged and turned into nonaggregated state. Since the $D$ value of dye DBos- 24 decreases by $40-50 \%$ after 600 minutes of irradiation, this dye is more photostable than Bos- 1 and DBos- 30 .

For the DNA+DBos-24 system, the optical density of aggregate band (Figure 9) decreases gradually with the irradiation time increasing but $D$ of nonaggregated dyes band (in presence of the DNA) remains constant. In our opinion, it is connected with the fact that nonaggregated dyes intercalate into the DNA macromolecule while aggregates bind outside the DNA chain. As it was mentioned above, during the irradiation the molecules of environmental oxygen are transferred in the singlet excited electronic state and become an active oxidant [30]. Generated singlet oxygen damages the aggregated dyes that are outside the DNA chain. The DNA screens nonaggregated dyes that are intercalated in the DNA macromolecule (between the DNA strands) and protect them from the influence of singlet oxygen and, therefore, the dyes molecules are not damaged.

As it is shown (Figure 8(b); curve 3), the increase (up to by $30 \%$ ) of $D$ of the DNA absorption spectra bands is observed for the DNA+DBos-24 system under the irradiation time increase. In our opinion, this phenomenon is caused by the untwisting or cutting of the strands of the DNA macromolecule which contacts with the molecules of DBos24 (this phenomenon is reverse to the hypochromic effect). These results agree with $37 \%$ increase for the complete DNA untwisting [34]. Thus the presence of DBos-24 dye causes the DNA damage, that is, DBos-24 showed phototoxic influence on the DNA. But dyes phototoxicity appears not only in the untwisting or cutting of the DNA strands. For the DNA+Dst-MdO system, the value of optical density of the DNA absorption band decreases by 10\% (Figure 10). We suppose it is connected with the direct DNA $\pi$-electron systems damage that is caused by Dst-MdO molecules.

So, DBos-24 and Dst-MdO dyes could be possibly used in the photodynamic therapy for the DNA damage.

\section{CONCLUSIONS}

Novel monomer and homodimer styrylcyanine dyes based on benzothiazole, naphtathiazole, benzoimidazole, pyridinium, quinoline, and 4-oxo-thieno[2,3-d]pyrimidinium heterocyclic residues were elaborated, synthesized, and studied. The dyes demonstrated rather high sensitivity to DNA.

Monomer and homodimer styrylcyanine dyes demonstrated fluorescence emission of high intensity upon TPE at wavelength of $840 \mathrm{~nm}$ and $1064 \mathrm{~nm}$. TPA cross sections of dyes at the wavelength $1064 \mathrm{~nm}$ are of the $10^{-50} \mathrm{~cm}^{4} \mathrm{~s}$ order. The dyes based on benzothiazole and benzoimidazole heterocycle generally demonstrate higher TPA cross sections values as compared to the other studied dyes.

Dyes with spermine-like linkage/tail groups demonstrated higher sensitivity to DNA and have higher TPA cross sections than dyes with the same chromophore but without respective groups.

We can conclude that the studies dyes can be used for SPE and TPE fluorescent DNA detection.

The developed dyes are rather photostable and mainly photochemicaly safe. The DBos-24 is most photostable but manifests some phototoxicity. The dye Dst-MdO is rather phototoxic; both these dyes could be possibly used in the photodynamic therapy.

\section{ACKNOWLEDGMENTS}

The authors are thankful to Dr. Tymish Y. Ohulchanskyy (Department of Chemistry and Institute for Lasers, Photonics and Biophotonics, SUNY at Buffalo, Buffalo, New York) for performing the fluorescence miscoscopy 
experiment. They are grateful to The National Academy of Science of Ukraine Center for Collective Use of "LASER FEMTOSECOND COMPLEX" attached to NASU Institute of Physics. The work was supported by the Science and Technology Center of Ukraine (grant no. U3104k).

\section{REFERENCES}

[1] R. P. Haugland, Handbook of Fluorescent Probes and Research Products, Molecular Probes, Eugene, Ore, USA, 9th edition, 2002.

[2] M. Albota, D. Beljonne, J.-L. Brédas, et al., "Design of organic molecules with large two-photon absorption cross sections," Science, vol. 281, no. 5383, pp. 1653-1656, 1998.

[3] D. J. S. Birch, "Multiphoton excited fluorescence spectroscopy of biomolecular systems," Spectrochimica Acta Part A, vol. 57, no. 11, pp. 2313-2336, 2001.

[4] D. Ramaiah, I. Eckert, K. T. Arun, L. Weidenfeller, and B. Epe, "Squaraine dyes for photodynamic therapy: mechanism of cytotoxicity and DNA damage induced by halogenated squaraine dyes plus light $(>600 \mathrm{~nm})$," Photochemistry and Photobiology, vol. 79, no. 1, pp. 99-104, 2004.

[5] J. A. Woods, N. J. Traynor, L. Brancaleon, and H. Moseley, "The effect of photofrin on DNA strand breaks and base oxidation in HaCaT keratinocytes: a comet assay study," Photochemistry and Photobiology, vol. 79, no. 1, pp. 105-113, 2004.

[6] R. Bersohn and I. Isenberg, "Phosphorescence in nucleotides and nucleic acids," The Journal of Chemical Physics, vol. 40, no. 11, pp. 3175-3180, 1964.

[7] D. Dee and M. E. Baur, "Charge and excitation migration in DNA chains," The Journal of Chemical Physics, vol. 60, no. 2, pp. 541-560, 1974.

[8] Yu. A. Vladimirov and V. N. Glagolev, "The study of the energy migration from the DNA on the acrydine orange dye," in Molecular Biophysics, G. M. Frank, Ed., Nauka, Moscow, Russia, 1965.

[9] R. G. Shulman and R. O. Rahn, "Electron spin resonance of the excited triplet states of pyrimidines and purines," The Journal of Chemical Physics, vol. 45, no. 8, pp. 2940-2946, 1966.

[10] R. O. Rahn, T. Yamane, J. Eisinger, J. W. Longworth, and R. G. Shulman, "Luminescence and electron spin resonance studies of adenine in various polynucleotides," The Journal of Chemical Physics, vol. 45, no. 8, pp. 2947-2954, 1966.

[11] W. Rhodes, "Hypochromism and other spectral properties of helical polynucleotides," Journal of the American Chemical Society, vol. 83, no. 17, pp. 3609-3617, 1961.

[12] J. W. Longworth, R. O. Rahn, and R. G. Shulman, "Luminescence of pyrimidines, purines, nucleosides, and nucleotides at $77^{\circ} \mathrm{k}$. The effect of ionization and tautomerization," The Journal of Chemical Physics, vol. 45, no. 8, pp. 2930-2939, 1966.

[13] A. A. Lamola, M. Guéron, T. Yamane, J. Eisinger, and R. G. Shulman, "Triplet state of DNA," The Journal of Chemical Physics, vol. 47, no. 7, pp. 2210-2217, 1967.

[14] M. Guéron, J. Eisinger, and R. G. Shulman, "Excited states of nucleotides and singlet energy transfer in polynucleotides," The Journal of Chemical Physics, vol. 47, no. 10, pp. 4077-4091, 1967.

[15] R. O. Rahn, R. G. Shulman, and J. W. Longworth, "Phosphorescence and electron spin resonance studies of the uv-excited triplet state of DNA," The Journal of Chemical Physics, vol. 45, no. 8, pp. 2955-2965, 1966.
[16] H. Fujita, M. Nakano, M. Takahata, and K. Yamaguchi, "A new strategy of enhancing two-photon absorption in conjugated molecules: introduction of charged defects," Chemical Physics Letters, vol. 358, no. 5-6, pp. 435-441, 2002.

[17] L.-Z. Wu, X.-J. Tang, M.-H. Jiang, and C.-H. Tung, "Twophoton induced fluorescence of novel dyes," Chemical Physics Letters, vol. 315, no. 5-6, pp. 379-382, 1999.

[18] V. B. Kovalska, D. V. Kryvorotenko, A. O. Balanda, M. Yu. Losytskyy, V. P. Tokar, and S. M. Yarmoluk, "Fluorescent homodimer styrylcyanines: synthesis and spectralluminescent studies in nucleic acids and protein complexes," Dyes and Pigments, vol. 67, no. 1, pp. 47-54, 2005.

[19] V. P. Tokar, M. Yu. Losytskyy, V. B. Kovalska, et al., "Fluorescence of styryl dyes-DNA complexes induced by single- and two-photon excitation," Journal of Fluorescence, vol. 16, no. 6, pp. 783-791, 2006.

[20] A. O. Balanda, K. D. Volkova, V. B. Kovalska, et al., "Synthesis and spectral-luminescent studies of novel 4-oxo-4,6,7,8tetrahydropyrrolo[1,2-a] thieno[2,3- $d]$ pyrimidinium styryls as fluorescent dyes for biomolecules detection," Dyes and Pigments, vol. 75, no. 1, pp. 25-31, 2007.

[21] A. O. Balanda, K. D. Volkova, V. B. Kovalska, M. Yu. Losytskyy, S. S. Lukashov, and S. M. Yarmoluk, "Novel styrylcyanines and their dimers as fluorescent dyes for nucleic acids detection: synthesis and spectral-luminescent studies," Ukrainica Bioorganica Acta, vol. 4, no. 1, pp. 17-29, 2006 (Ukrainian).

[22] S. M. Yarmoluk, M. Yu. Losytskyy, and V. M. Yashchuk, "Nonradiative deactivation of the electronic excitation energy in cyanine dyes: influence of binding to DNA," Journal of Photochemistry and Photobiology B, vol. 67, no. 1, pp. 57-63, 2002.

[23] M. Yu. Losytskyy, N. Akbay, V. B. Kovalska, A. O. Balanda, and S. M. Yarmoluk, "Study of styryl dyes-dsDNA binding equilibrium," in Proceedings of the 1st Asian Spectroscopy Conference and Asian Biospectroscopy Conference, Book of Abstracts, pp. 206-207, Bangalore, India, January-February 2007.

[24] S. M. Yarmoluk, S. S. Lukashov, M. Yu. Losytskyy, B. Akerman, and O. S. Kornyushyna, "Interaction of cyanine dyes with nucleic acids: XXVI. Intercalation of the trimethine cyanine dye Cyan 2 into double-stranded DNA: study by spectral luminescence methods," Spectrochimica Acta Part A, vol. 58, no. 14, pp. 3223-3232, 2002.

[25] H. S. Rye, M. A. Quesada, K. Peck, R. A. Mathies, and A. N. Glazer, "High-sensitivity two-color detection of doublestranded DNA with a confocal fluorescence gel scanner using ethidium homodimer and thiazole orange," Nucleic Acids Research, vol. 19, no. 2, pp. 327-333, 1991.

[26] D. J. Bradley, M. H. R. Hutchinson, and H. Koetser, "Interactions of picosecond laser pulses with organic molecules. II. Two-photon absorption cross-section," Proceedings of the Royal Society of London. Series A, vol. 329, no. 1576, pp. 105119, 1972.

[27] T. Yu. Ogul'chansky, V. M. Yashchuk, M. Yu. Losytskyy, I. O. Kocheshev, and S. M. Yarmoluk, "Interaction of cyanine dyes with nucleic acids. XVII. Towards an aggregation of cyanine dyes in solutions as a factor facilitating nucleic acid detection," Spectrochimica Acta Part A, vol. 56, no. 4, pp. 805-814, 2000.

[28] V. Yashchuk, V. Kudrya, M. Losytskyy, H. Suga, and T. Yu. Ohul'chanskyy, "The nature of the electronic excitations capturing centres in the DNA," Journal of Molecular Liquids, vol. 127, no. 1-3, pp. 79-83, 2006.

[29] V. M. Yashchuk, V. Yu. Kudrya, M. Yu. Losytskyy, et al., "The effect of triplet-triplet excitation energy transfer on the DNA 
self-protection mechanism," The Scientific Notes of NaUKMA: Physics, vol. 51, pp. 48-56, 2006.

[30] D. G. Knorre, O. S. Fedorova, and E. I. Frolova, "The oxidation degradation of nucleic acids," Chemical Progress, vol. 62, no. 1, pp. 70-91, 1993 (Russian).

[31] A. A. Ishchenko, The Structure and Spectral Luminescent Properties of Polymethine Dyes, Naukova Dumka, Kyiv, Ukrania, 1994.

[32] H. S. Rye, S. Yue, D. E. Wemmer, et al., "Stable fluorescent complexes of double-stranded DNA with bis-intercalating asymmetric cyanine dyes: properties and applications," Nucleic Acids Research, vol. 20, no. 11, pp. 2803-2812, 1992.

[33] H. S. Rye and A. N. Glazer, "Interaction of dimeric intercalating dyes with single-stranded DNA," Nucleic Acids Research, vol. 23, no. 7, pp. 1215-1222, 1995.

[34] D. Freifelder, Physical Biochemistry. Applications to Biochemistry and Molecular Biology, W.H. Freeman, San Francisco, Calif, USA, 1976.

[35] T. Yu. Ogul'chansky, M. Yu. Losytskyy, V. B. Kovalska, V. M. Yashchuk, and S. M. Yarmoluk, "Interactions of cyanine dyes with nucleic acids. XXIV. Aggregation of monomethine cyanine dyes in presence of DNA and its manifestation in absorption and fluorescence spectra," Spectrochimica Acta Part A, vol. 57, no. 7, pp. 1525-1532, 2001.

[36] W. West and S. Pearce, "The dimeric state of cyanine dyes," Journal of Physical Chemistry, vol. 69, no. 6, pp. 1894-1903, 1965. 

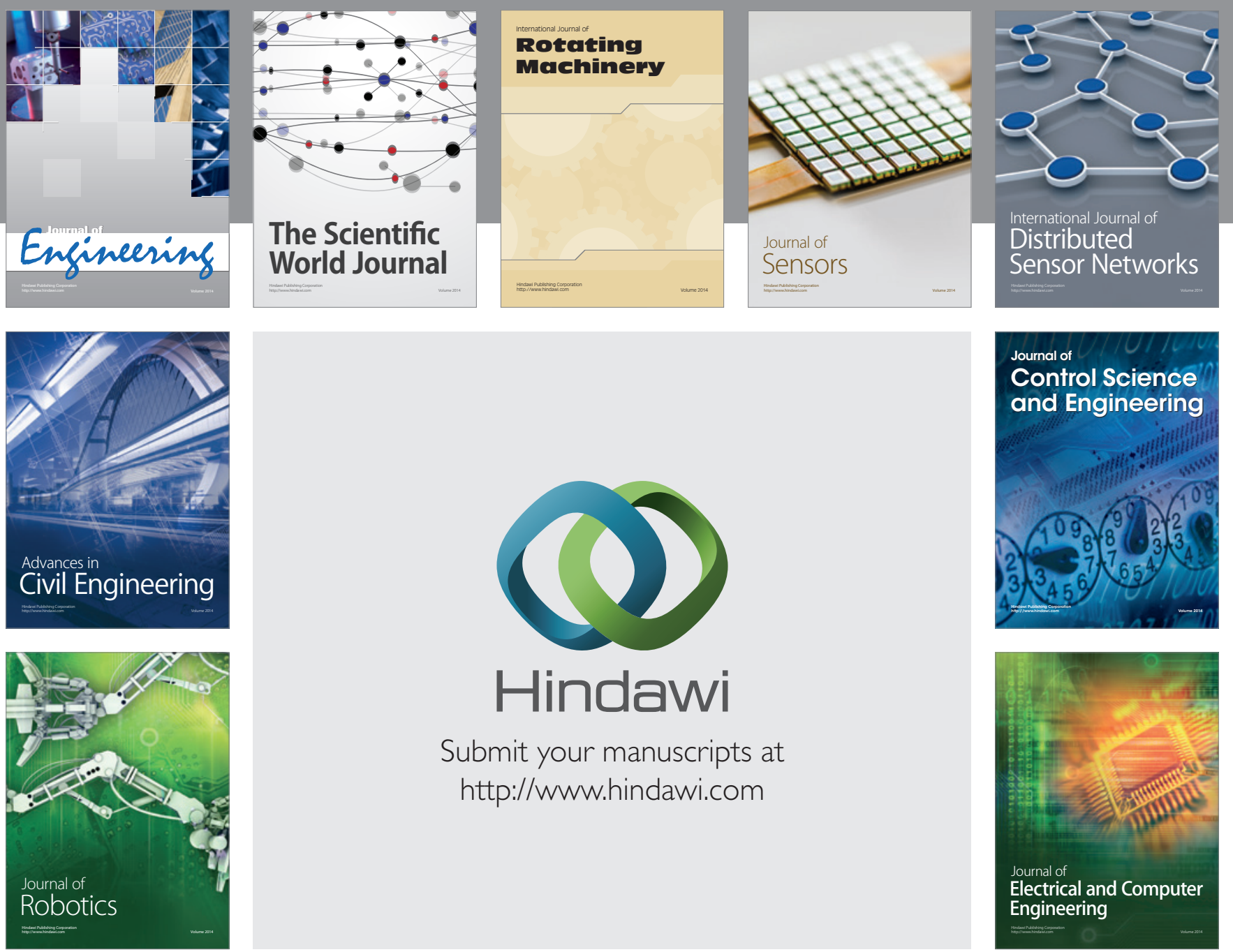

Submit your manuscripts at

http://www.hindawi.com
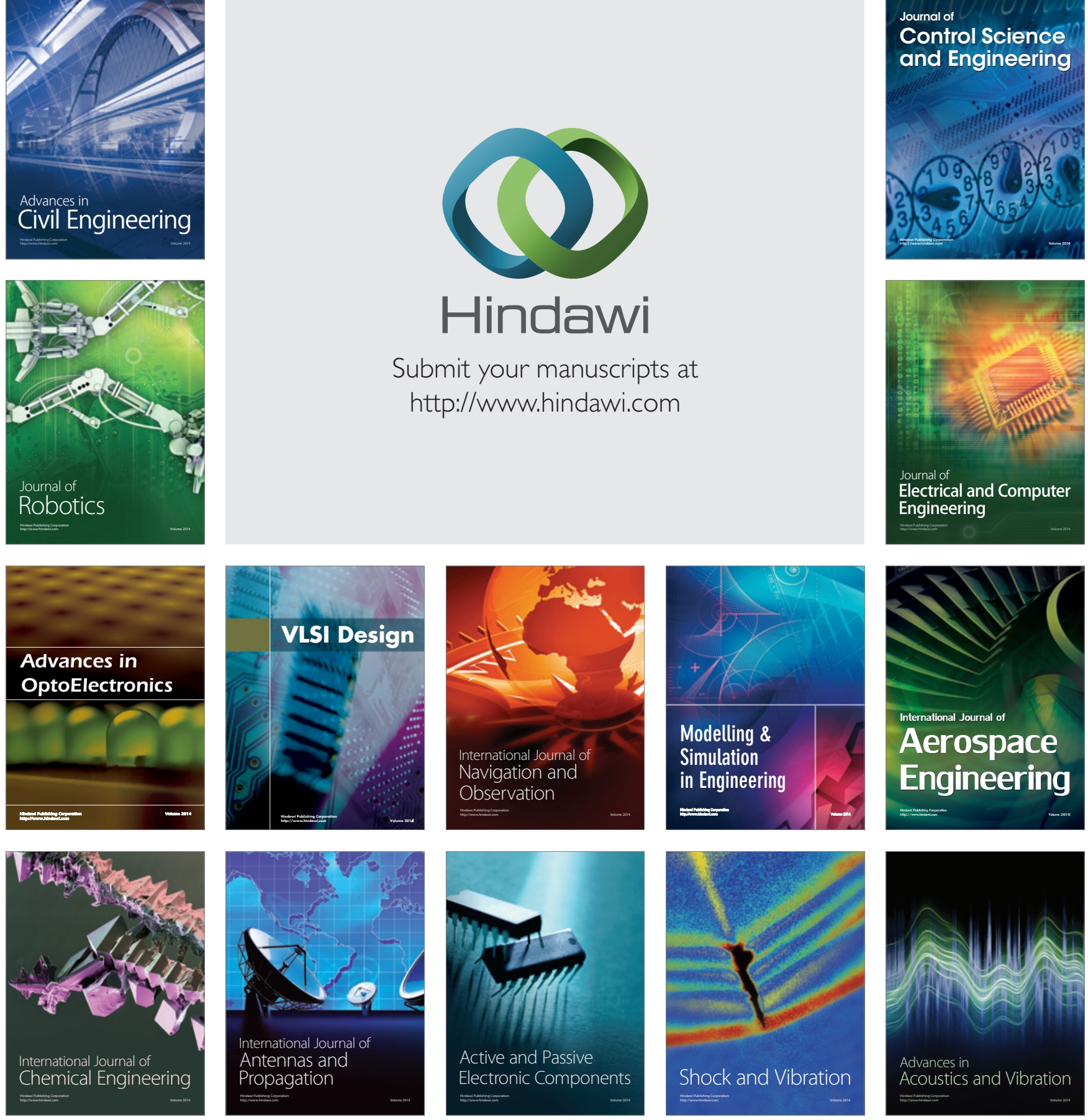